\title{
Neonatal mortality in the urban and rural China between 1996 and 2013: a retrospective study
}

\author{
Ruifeng Lu ${ }^{1,2}$, Xiaohong Li ${ }^{3}$, Sufang Guo ${ }^{4}$, Huiqing Wang ${ }^{1,2}$, Jun Zhu ${ }^{3}$, Chunhua He ${ }^{3}$, Qi Li ${ }^{3}$, Leni Kang ${ }^{3}$, Yanping Wang ${ }^{3}$, Jun Tang ${ }^{1}$ \\ and Dezhi $\mathrm{Mu}^{1}$
}

BACKGROUND: One of the proposed United Nations post2015 sustainable development goals is to eliminate avoidable neonatal deaths by 2030. This study aims to examine changes in neonatal mortality rate (NMR) and cause-specific NMR in urban and rural areas to guide renewed efforts to further reduce NMR in China.

METHODS: This retrospective study used 18 y of consecutive data from the National Under-5 Child Mortality Surveillance System.

RESULTS: Urban NMR decreased from 11.0 to 4.0 deaths per 1,000 live births, and rural NMR was decreased births from 26.0 to 8.1 deaths per 1,000 live births between the periods of 1996-1998 and 2011-2013. Rural NMR was about two times as much as urban NMR in 2011-2013. Approximately four-fifths of neonatal deaths occurred within less than $7 \mathrm{~d}$ after birth. In 2011-2013, the three leading causes of early neonatal death were premature delivery, intrapartum-related conditions, and congenital abnormalities; the three causes of death in late neonates were classification premature delivery, congenital abnormalities, and pneumonia.

CONCLUSION: China has made substantive progress in reducing NMR; however, the disparity in NMR between urban and rural areas still exists. More effort should be made to prevent and manage premature delivery and congenital abnormalities, especially in rural areas.

M illennium Development Goal 4 has encouraged many countries to make substantial efforts to reduce child morality. Child survival has extremely improved. Although the health of children under 5 has substantially improved globally, with the number of children under- 5 deaths decreasing from 17.6 million in 1970 to 6.3 million in 2013, only 27 developing countries are expected to achieve Millennium Development Goal 4 by 2015 (1). Moreover, declines in neonatal mortality are not keeping pace with declines in postneonatal mortality, which has globally increased the burden of newborn death.
From 1990 to 2007, the under-5 mortality rate in China decreased by over $66.7 \%$, which was 8 y ahead of the deadline imposed by Millennium Development Goal 4 (2). Previous studies have described the rapid fall in child mortality in China and have shown the important role that socioeconomic and health system determinants have had in the process $(3,4)$. However, neonatal deaths in China accounted for over $50 \%$ of children under- 5 deaths (5) and 5\% of global newborn deaths, which will largely influence the global burden of disease. Because of differences in social development in urban and rural China, large rural-urban disparities in neonatal mortality rate (NMR) in China have been shown in a previous study (6). The change in NMR and cause-specific NMR in urban and rural areas provide an important geographical summary of progress in reducing the burden of newborn death and illustrate where renewed efforts are needed. Currently, there are no published data to show cause-specific NMR in urban and rural areas.

This study aims to understand the change in urban and rural NMR, as well as changes in cause-specific NMR, from 1996 to 2013. The study also aims to assess the urban-rural disparity in NMR using $18 \mathrm{y}$ of consecutive data from the populationbased National Under-5 Child Mortality Surveillance System (NU5CMSS). With this, progress and challenges in neonatal health can be identified and provide direction for the Chinese government to create effective interventions that improve neonatal health. Additionally, useful lessons can be gleaned from the experience of reducing neonatal mortality in China and transferred to developing countries across the world.

\section{RESULTS}

Table 1 shows that the NMR in urban areas was decreased from 11.0 to 4.0 deaths per 1,000 live births, which was a 63.8\% decrease between the periods of 1996-1998 and 20112013. The early neonatal deaths accounted for $79.1 \%$ of all neonatal deaths, and the urban early neonatal mortality rate (ENMR) was reduced by $67.4 \%$. The urban NMRs caused by

\footnotetext{
The first two authors are joint first authors

'Department of Pediatrics, West China Second University Hospital, Sichuan University, Chengdu, Sichuan, China; ${ }^{2}$ Key Laboratory of Obstetric and Gynecologic and Pediatric Diseases and Birth Defects of Ministry of Education, West China Second University Hospital, Sichuan University, Chengdu, Sichuan, China; ${ }^{3}$ National Office for Maternal and Child Health Surveillance, West China Second University Hospital, Sichuan University, Chengdu, Sichuan, China; ${ }^{4}$ UNICEF Office for China, Beijing, China.

Correspondence: Jun Tang (tj1234753@sina.com)

Received 12 March 2015; accepted 2 November 2015; advance online publication 17 February 2016. doi:10.1038/pr.2016.6
} 


\section{Articles | Luetal.}

Table 1. The urban-rural-specific neonatal mortality rates (per 1,000 live births) in China between 1996 and 2013

\begin{tabular}{lcrrrrrr}
\hline & & \multicolumn{2}{c}{ Early } & \multicolumn{2}{c}{ Late } & \multicolumn{2}{c}{ Total } \\
\cline { 3 - 8 } Area & Periods & $N$ & ENMR & $N$ & LNMR & $N$ & NMR \\
\hline Urban & $1996-1998$ & 1,465 & 9.2 & 279 & 1.8 & 1,744 & 11.0 \\
& $1999-2001$ & 1,356 & 8.4 & 363 & 2.2 & 1,718 & 10.6 \\
& $2002-2004$ & 1,199 & 7.3 & 290 & 1.8 & 1,489 & 9.0 \\
& $2005-2007$ & 976 & 4.6 & 231 & 1.1 & 1,207 & 5.7 \\
& $2008-2010$ & 1,277 & 3.3 & 410 & 1.1 & 1,687 & 4.3 \\
& $2011-2013$ & 1,622 & 3.0 & 522 & 1.0 & 2,143 & 4.0 \\
& CA (\%) & - & -67.4 & - & -45.0 & - & -63.8 \\
\hline Rural & $1996-1998$ & 3,024 & 20.3 & 845 & 5.7 & 3,869 & 26.0 \\
& $1999-2001$ & 2,811 & 20.9 & 754 & 5.6 & 3,565 & 26.6 \\
& $2002-2004$ & 2,239 & 15.4 & 614 & 4.2 & 2,853 & 19.6 \\
& $2005-2007$ & 1,886 & 11.8 & 452 & 2.8 & 2,338 & 14.7 \\
& $2008-2010$ & 4,257 & 8.5 & 1,205 & 2.4 & 5,462 & 10.9 \\
& $2011-2013$ & 4,463 & 6.3 & 1,328 & 1.9 & 5,791 & 8.1 \\
CA (\%) & - & -69.2 & - & -67.2 & - & -68.7 \\
\hline
\end{tabular}

CA, changing amplitude; NMR, neonatal mortality rate; ENMR, early neonatal mortality rate; LNMR, late neonate mortality rate.

noninfectious and infectious diseases were reduced by 63.1 and $65.1 \%$ during the study periods, respectively. The causes of child deaths that were absolutely reduced the most were intrapartum-related conditions, congenital abnormalities, premature delivery, and pneumonia. The decline of these causespecific NMRs contributes to the $92.5 \%$ reduction of urban NMR (Table 2).

In rural areas, the NMR decreased from 26.0 to 8.1 deaths per 1,000 live births, which was a $68.7 \%$ decrease. Of all neonatal deaths, $78.2 \%$ occurred within less than $7 \mathrm{~d}$ after birth. The rural ENMR was reduced by $69.2 \%$ between the periods of 1996-1998 and 2011-2013 periods (Table 1). The noninfectious disease-specific NMR was reduced by $66.4 \%$ during the same period. This was mainly due to a decreased mortality in intrapartum-related conditions and preterm delivery. Meanwhile, infectious disease-specific NMR was reduced by $81.6 \%$, and approximately $78.9 \%$ of that reduction was due to the decline of the child mortality caused by pneumonia.

The risk ratio (RR) of NMR between rural and urban areas decreased from 2.46 (95\% confidence interval (CI): 2.32-2.60) in 1996-1998 to 2.04 (95\% CI: $1.94-2.14$ ) in 2011-2013. However, until 2011-2013, almost all cause-specific NMRs in rural areas were significantly higher than those in urban areas, except for measles, septicemia, meningitis, and tetanus (Table 2). Notably, the NMR due to premature delivery that has become the leading cause of child death in 2011-2013 in rural areas was about two times as much as that in urban areas.

Almost all cause-specific ENMRs substantially decreased both in urban and rural areas of China over the last $18 \mathrm{y}$ (Table 3). Of note, pneumonia-specific ENMR decreased by more than three-quarter during the study periods, as well as the ENMR due to intrapartum-related conditions. However, the proportion of early neonatal deaths due to premature delivery significantly increased from $22.9 \%$ in $1996-1998$ to $36.2 \%$ in 2011-2013 in urban areas $(Z=11.62, P<0.001)$ and from $26.4 \%$ in $1996-1998$ to $36.3 \%$ in $2011-2013$ in rural areas $(Z=10.58, P<0.001)$. The proportion of early neonatal deaths caused by congenital abnormalities increased from $9.2 \%$ during $1996-1998$ to $16.5 \%$ during $2011-2013$ in rural areas $(Z=$ $12.03, P<0.001)$, but no significant change was observed in urban areas (Figure 1). In 2011-2013, the three leading causes of early neonatal deaths were premature delivery, intrapartumrelated conditions, and congenital abnormalities, and the three leading causes of late neonatal death were premature delivery, congenital abnormalities, and pneumonia (Figure 1; Table 3).

\section{DISCUSSION}

Our study indicates a remarkable decline in the NMR both in urban and rural areas of China over the past $18 \mathrm{y}$; the percent decrease was more than $60 \%$, much faster than the global average (1). The NMR of urban China in 2011-2013 approaches that of some developed countries or regions, such as Canada $(3.1 \%)$ ), Central Europe $(3.4 \%)$, and America (3.6\%). The NMR of rural China is also much lower than the global NMR $(20 \%)$ and many middle income and developing countries, such as Brazil (9.1\%o) and India (28.1\%o) (1). It is impossible to achieve MGD4 without substantial reductions in early neonatal mortality (7). Estimates indicate that global ENMR was reduced by $37.6 \%$, which was much lower than late neonatal mortality rate (52.7\%) from 1990 to 2013 (1). Our study shows substantial decreases in ENMR in both urban and rural areas; the changing amplitude of ENMR was more than two-thirds from 1996-1998 to 2011-2013. China's success suggests that reducing the ENMR is critical to reducing child mortality.

A substantial reduction of the NMR due to infectious diseases was observed in our study. This was facilitated by having full access to antibiotics (oral medication and intravenous drip), the promotion of breast feeding, the practice of boiling water, and the presence of a skilled birth attendant $(4,8,9)$. More importantly, the implementation of several important public health programs by the Chinese government has also contributed largely to this achievement. The coverage of sanitary latrines in rural areas increased from 39.8\% in 1999 to $71.7 \%$ in 2012 , and the coverage of clean water supplies increased from $43.2 \%$ in 1995 to $74.6 \%$ in 2012 . These projects benefited from the program for improving drinking water and lavatories initiated in 1990s (5). Moreover, the programs of Reducing Maternal Mortality and Eliminate Neonatal Tetanus initiated in 2000 (expanded to 2,218 counties/districts in 2012 from only the 378 poorest counties in 2000) and Integrated Management of Childhood Illness initiated in 2003 (expanded to 22 provinces in 2008 from 2 provinces in 2003) have had a positive impact on the control of these infectious diseases (10-12).

Additionally, our findings suggest that the reduction of NMR in both urban and rural areas is largely attributed to decreased neonatal mortality of intrapartum-related conditions, premature delivery, and congenital abnormalities. The 
Table 2. The cause-specific neonatal mortality rates (per 100,000 live birth) in urban and rural China between 1996 and 2013

\begin{tabular}{|c|c|c|c|c|c|}
\hline \multirow[b]{2}{*}{ Conditions } & \multirow[b]{2}{*}{ Periods } & \multicolumn{2}{|c|}{ NMR $(95 \% \mathrm{Cl})$} & \multicolumn{2}{|c|}{$\mathrm{RR}(95 \% \mathrm{Cl})$} \\
\hline & & Urban & Rural & Urban & Rural \\
\hline \multirow[t]{4}{*}{ Noninfectious diseases } & 1996-1998 & $947.6(900.7-996.6)$ & $2,107.4(2,035.0-2,182.5)$ & 1 & $2.22(2.09-2.37)$ \\
\hline & 2002-2004 & $818.7(775.9-863.3)$ & $1,679.9(1,614.5-1,747.6)$ & 1 & $2.05(1.92-2.19)$ \\
\hline & 2005-2007 & $597.7(565.5-631.5)$ & $1,189.2(1,136.6-1,243.7)$ & 1 & $1.99(1.85-2.14)$ \\
\hline & CA (\%) & -63.1 & -66.4 & & \\
\hline \multirow[t]{4}{*}{ Premature delivery } & 1996-1998 & $229.0(206.6-253.8)$ & $640.7(601.2-682.6)$ & 1 & $2.80(2.48-3.16)$ \\
\hline & 1999-2001 & $243.0(220.2-268.4)$ & $663.4(620.9-708.1)$ & 1 & $2.73(2.42-3.07)$ \\
\hline & $2002-2004$ & $206.0(184.9-228.8)$ & $581.7(543.6-621.9)$ & 1 & $2.80(2.49-3.21)$ \\
\hline & CA (\%) & -40.5 & -58.2 & & \\
\hline \multirow[t]{7}{*}{ Intrapartum-related conditions } & 1996-1998 & $404.8(374.8-437.5)$ & $798.3(754.5-845.3)$ & 1 & $1.97(1.79-2.17)$ \\
\hline & 1999-2001 & $331.0(304.1-360.2)$ & $774.3(728.5-822.7)$ & 1 & $2.34(2.11-2.60)$ \\
\hline & 2002-2004 & $340.7(313.9-370.4)$ & $622.3(583.3-664.4)$ & 1 & $1.83(1.64-2.03)$ \\
\hline & 2005-2007 & $210.3(191.6-230.7)$ & $381.7(352.5-413.2)$ & 1 & $1.82(1.61-2.05)$ \\
\hline & $2008-2010$ & $113.6(103.6-124.8)$ & $304.7(289.8-320.3)$ & 1 & $2.68(2.41-2.98)$ \\
\hline & $2011-2013$ & $97.2(89.3-105.9)$ & 199.0 (188.9-209.6) & 1 & $2.05(1.85-2.26)$ \\
\hline & CA (\%) & -76.0 & -75.1 & & \\
\hline \multirow[t]{2}{*}{ Congenital abnormalities } & 1996-1998 & $233.1(210.2-257.8)$ & $250.3(225.9-276.8)$ & 1 & $1.07(0.93-1.24)$ \\
\hline & CA (\%) & -66.1 & -42.9 & & \\
\hline \multirow[t]{7}{*}{ Infectious diseases } & 1996-1998 & $140.3(122.8-159.7)$ & $569.2(532.3-609.1)$ & 1 & $4.07(3.51-4.71)$ \\
\hline & 1999-2001 & $97.7(83.6-114.2)$ & $500.7(464.2-540.0)$ & 1 & $5.12(4.31-6.09)$ \\
\hline & 2002-2004 & $74.7(62.5-89.1)$ & $337.8(309.4-369.2)$ & 1 & $4.53(3.72-5.52)$ \\
\hline & 2005-2007 & $60.1(50.5-71.6)$ & $202.4(181.2-225.5)$ & 1 & $3.36(2.74-4.13)$ \\
\hline & 2008-2010 & $49.3(42.7-56.8)$ & $140.1(130.2-150.9)$ & 1 & $2.85(2.43-3.34)$ \\
\hline & $2011-2013$ & $49.0(43.5-55.4)$ & $104.5(97.2-112.2)$ & 1 & $2.13(1.85-2.45)$ \\
\hline & CA (\%) & -65.1 & -81.6 & & \\
\hline \multirow[t]{7}{*}{ Pneumonia } & 1996-1998 & $117.7(102.2-136.1)$ & $450.2(417.6-485.8)$ & 1 & $3.82(3.25-4.49)$ \\
\hline & 1999-2001 & $79.1(66.6-94.2)$ & $404.1(371.2-439.3)$ & 1 & $5.10(4.21-6.18)$ \\
\hline & 2002-2004 & $60.0(49.3-73.1)$ & $284.5(258.4-313.3)$ & 1 & $4.74(3.80-5.90)$ \\
\hline & 2005-2007 & $50.5(41.9-61.2)$ & $165.1(146.3-186.3)$ & 1 & $3.26(2.60-4.08)$ \\
\hline & $2008-2010$ & $34.5(29.2-40.9)$ & $111.0(102.3-120.7)$ & 1 & $3.22(2.66-3.88)$ \\
\hline & $2011-2013$ & $33.8(29.3-39.1)$ & $83.7(77.2-90.6)$ & 1 & $2.47(2.09-2.92)$ \\
\hline & CA (\%) & -71.3 & -81.4 & & \\
\hline
\end{tabular}




\section{Articles | Luetal.}

Table 2. Continued

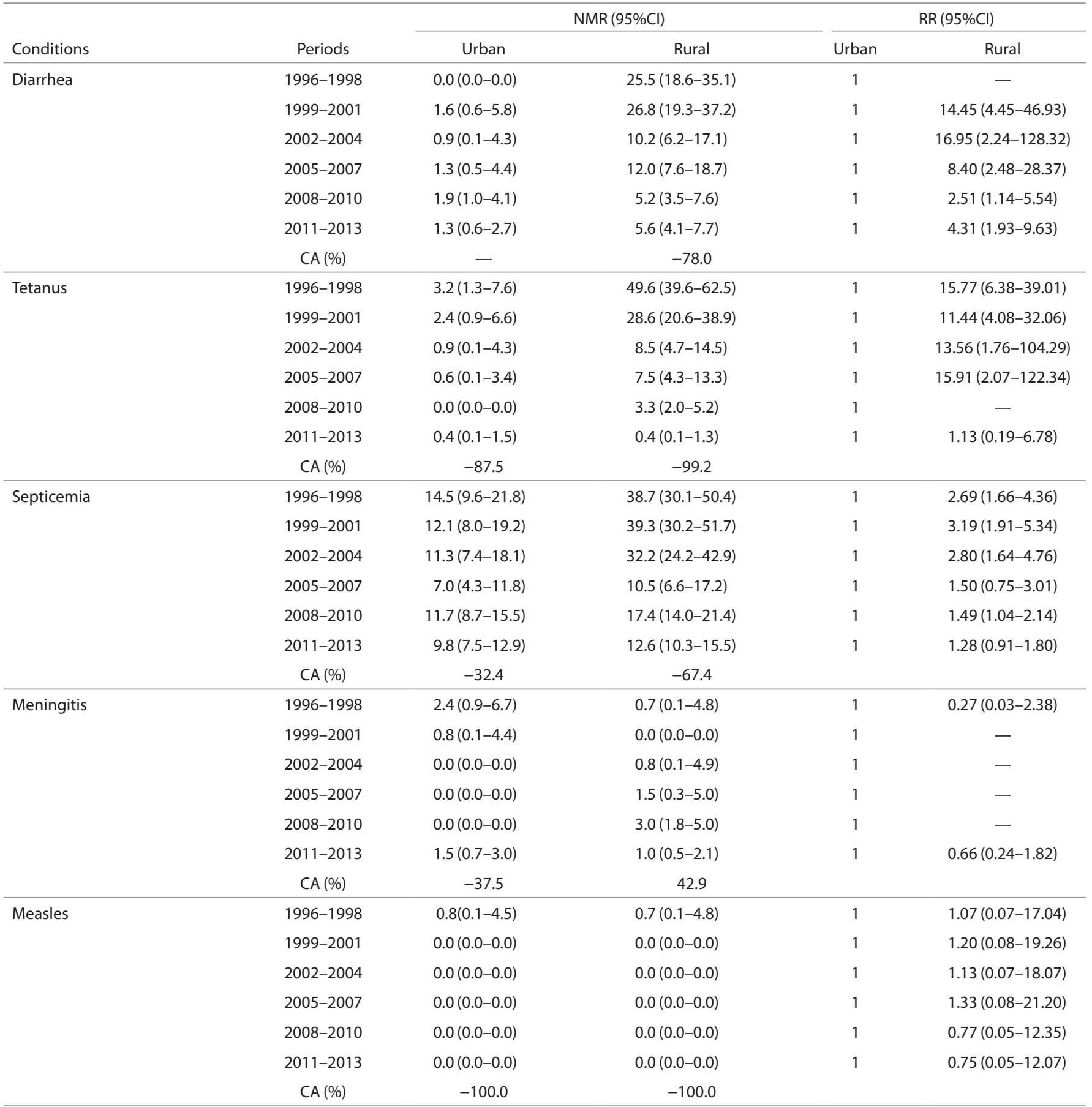

CA, changing amplitude.

decreases in these cause-specific NMRs may also be due to increased accessibility of health services and improvement of skilled health care before, during, and after delivery, as well as the impact of neonatal resuscitation and the policy that at least one staff member trained in neonatal resuscitation is present in labor room. In the past $18 \mathrm{y}$, through the use of in-facility delivery subsidy for rural women and a wide range of health education $(10,13)$, the in-facility delivery rate increased from $60.7 \%$ in 1996 to $99.5 \%$ in 2013 (14). Previous studies suggest that $48-70 \%$ of neonate deaths could have been prevented by hospital birth (13). In view of the fact that intrapartum-related conditions were the leading cause of child deaths in China before 2004, the intervention package promoting effective neonatal resuscitation training at county-level hospitals initiated in 2004 by Health Ministry of China, covering 20 central and western provinces. In the same year, the Department of Maternal and Child Health ( $\mathrm{MCH})$ Care and Community Health of Health Ministry released the guideline for neonatal 
Table 3. The cause-specific early and late neonatal mortality rates (per 100,000 live birth) in urban and rural China between 1996 and 2013

\begin{tabular}{|c|c|c|c|c|c|c|c|}
\hline \multirow[b]{2}{*}{ Neonate } & \multirow[b]{2}{*}{ Conditions } & \multicolumn{3}{|c|}{ Urban } & \multicolumn{3}{|c|}{ Rural } \\
\hline & & 1996-1998 & 2011-2013 & CA (\%) & 1996-1998 & 2011-2013 & CA (\%) \\
\hline \multirow[t]{9}{*}{ Early neonate } & Premature delivery & 208.9 & 109.1 & -47.8 & 552.4 & 227.6 & -58.8 \\
\hline & Intrapartum-related conditions & 364.5 & 86.3 & -76.3 & 778.6 & 184.8 & -76.3 \\
\hline & Congenital abnormalities & 186.3 & 56.0 & -70.0 & 192.6 & 103.3 & -46.4 \\
\hline & Other noninfectious diseases & 62.9 & 23.2 & -63.1 & 293.3 & 57.8 & -80.3 \\
\hline & Pneumonia & 81.4 & 20.0 & -75.5 & 232.0 & 45.1 & -80.5 \\
\hline & Septicemia & 7.3 & 4.8 & -34.3 & 17.5 & 5.1 & -71.1 \\
\hline & Meningitis & 0.8 & 0.2 & -73.1 & 0.0 & 0.4 & - \\
\hline & Measles & 0.0 & 0.0 & - & 0.0 & 0.0 & - \\
\hline & Other infectious diseases & 0.8 & 1.5 & 88.3 & 1.5 & 0.8 & -46.7 \\
\hline \multirow[t]{8}{*}{ Late neonate } & Premature delivery & 20.2 & 27.1 & 34.5 & 88.3 & 40.5 & -54.2 \\
\hline & Pneumonia & 36.3 & 13.9 & -61.7 & 218.2 & 38.5 & -82.3 \\
\hline & Diarrhea & 0.0 & 1.1 & - & 18.2 & 4.5 & -75.5 \\
\hline & Tetanus & 2.4 & 0.2 & -91.0 & 32.8 & 0.2 & -99.4 \\
\hline & Septicemia & 7.3 & 5.0 & -31.3 & 21.2 & 7.6 & -64.1 \\
\hline & Meningitis & 1.6 & 1.3 & -19.3 & 0.7 & 0.6 & -20.0 \\
\hline & Measles & 0.8 & 0.0 & -100.0 & 0.7 & 0.0 & -100.0 \\
\hline & Other infectious diseases & 0.8 & 0.7 & -19.3 & 2.2 & 0.4 & -82.2 \\
\hline
\end{tabular}

CA, changing amplitude.

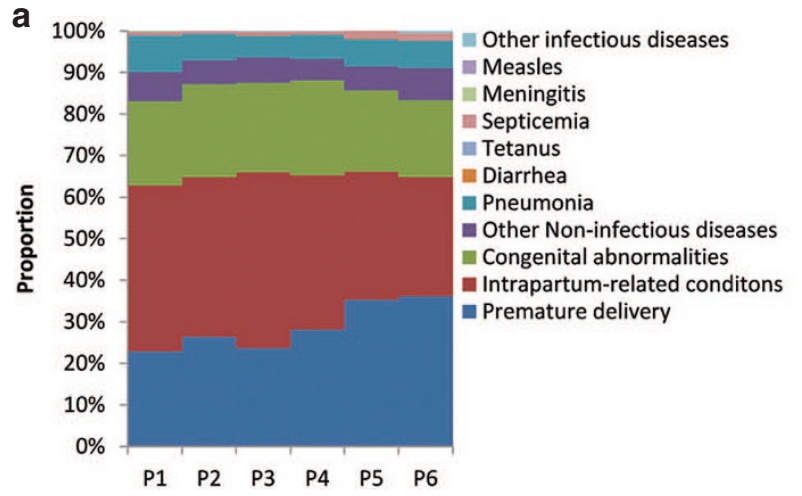

C

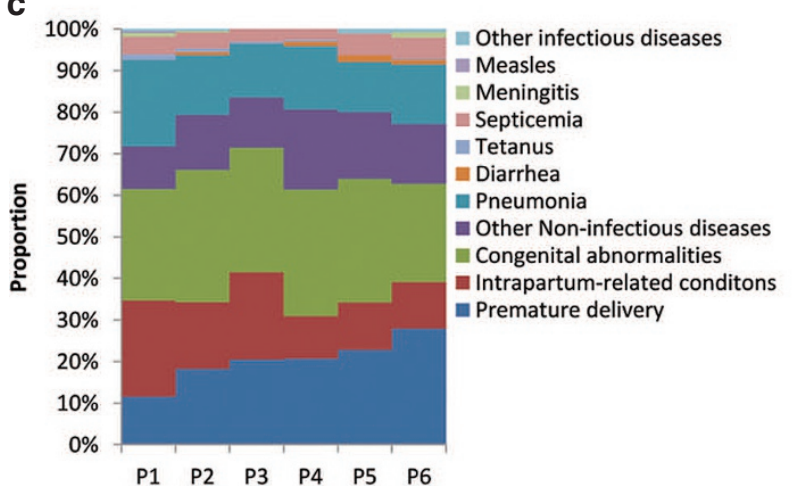

b

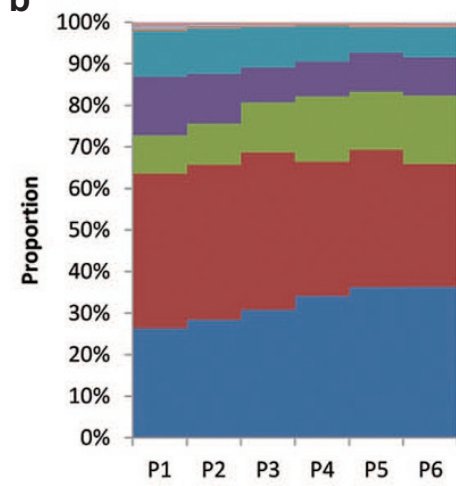

d

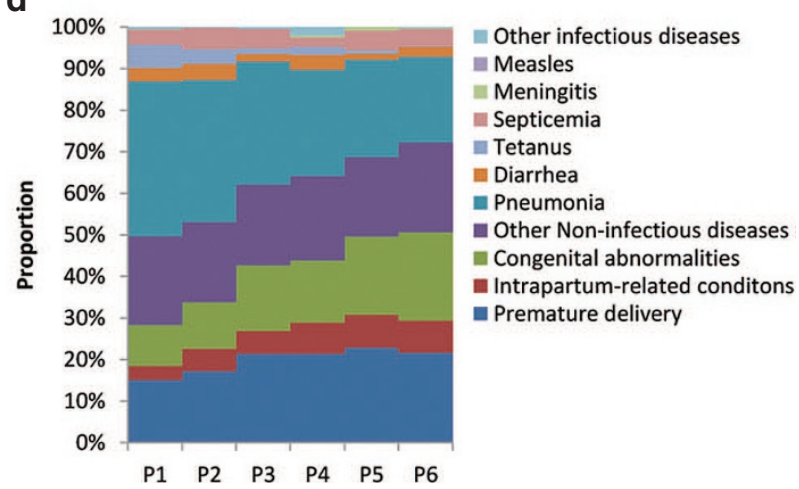

Figure1. Proportion of neonatal deaths due to different causes in urban and rural areas of China, by periods (P1: 1996-1998, P2: 1999-2001, P3: 2002-2004, P4: 2005-2007, P5: 2008-2010, P6: 2011-2013). (a) Proportion of early neonatal deaths due to different causes in urban areas of China; (b) proportion of early neonatal deaths due to different causes in rural areas of China; (c) proportion of late neonatal deaths due to different causes in urban areas of China; and (d) proportion of late neonatal deaths due to different causes in rural areas of China. 


\section{Articles | Luetal.}

resuscitation. These interventions have improved the skills of health providers and contribute to decrease the mortality of birth asphyxia (15). Our study shows that intrapartum-related conditions are no longer the leading cause of neonatal deaths in China as of 2011-2013.

However, because of relatively lower changing amplitude of the NMR due to premature delivery than that of the NMR due to intrapartum-related conditions in both urban and rural areas of China, premature delivery becomes the leading cause for neonatal deaths. The more difficulty of premature complication intervention and large number of preterm birth (1.17 million per year in China estimated by World Health Organization) in China may contribute to the increasing proportion of deaths due to premature delivery. Premature delivery accounts for approximately $36 \%$ of all early neonatal deaths and $33 \%$ of all neonatal deaths in 2011-2013. In the USA, only 17\% of neonatal deaths were due to preterm birth (16). Therefore, more attention should be given to reduce neonatal mortality caused by preterm delivery. Essential neonatal care, antenatal steroid injections, kangaroo mother care, and antibiotics to treat newborn infections are all feasible, cost-effective interventions to address preterm birth (17-20). Additionally, public investments should been made for the creation of neonatal intensive care units throughout the country and for strengthening basic and comprehensive essential newborn care, especially rural areas $(21,22)$. An estimated 276,000 babies worldwide die within 4 wk of birth each year from congenital abnormalities (23). Our results suggest that congenital abnormalities have emerged as important causes of neonatal mortality in rural areas as neonatal mortality caused by infectious diseases have declined. Reducing the incidence and mortality rate of congenital abnormalities, especially in rural areas, should be a priority for neonatal health in the future. The Chinese government should strive to increase the coverage of the prevention and treatment interventions for congenital abnormalities suggested by the World Health Organization (24).

Notably, our study also shows the disparity in NMR between urban and rural areas of China. Although rural-urban disparities in neonatal mortality risk caused by septicemia and tetanus have been eliminated, the rural NMR due to the noninfectious disease is significantly higher than the urban NMR. Unbalanced development of the economy and disparities in the accessibility of high-quality health care may contribute to the unfair neonatal survival in these two environments $(3,6,25)$. Moreover, we should note that the NMR due to infectious diseases, such as pneumonia and diarrhea, in rural areas is more than twice that of urban areas. Further action to reduce pneumonia and diarrhea incidence should involve the introduction of polyvalent pneumococcal, Hib, and rotavirus vaccines, which are still not freely provided to children, and the use of oral rehydration salts and zinc, which are still not a standard practice in China $(26,27)$.

There are some limitations to our study. First, misclassification of the cause of death is likely happened in NU5CMSS, especially for the deaths that occurred at home, which accounted for $32.7 \%$ of all deaths registered in NU5CMSS in
2013. However, $86.9 \%$ of the cases received medical treatment before death; therefore, we can easily obtain information about these deaths and identify the causes. Second, neonate deaths due to other causes accounted for $10.8 \%$ of all neonate deaths from 1996 to 2013 and need to redefined. Efforts have been made to improve the cause of death classification based on international standards, verbal autopsy in children found dead at home and neonatal death audits are the most direct methods for improving data collection on cause of death and child mortality estimation.

In summary, China has made a substantive progress in reducing NMR in both urban and rural areas over the past $18 \mathrm{y}$; however, disparities in NMR between urban and rural areas still exist. One of United Nations post-2015 sustainable development goals is to eliminate avoidable neonatal deaths by 2030. To further reduce child mortality, the Chinese government needs to make a stronger effort in training $\mathrm{MCH}$ professionals, improving the quality of neonatal care and access to quality essential obstetric and neonatal services, promoting prenatal health education and financially supporting women and children in poor areas.

\section{METHODS}

\section{NU5CMSS in China}

Data used in this study were obtained from NU5CMSS, a well-established population-based under-5 child death registry system set up by Health Ministry of China in 1991. There were 116 districts/counties sampled in this system. These sites were spread across 31 provinces, autonomous regions, and municipalities from 1996 to 2008. The number of surveillance sites increased to 336 districts/counties in 2009, covering a population of approximately 36 million (3\% of total population). According to the women's residence, the surveillance sites can be classified into urban areas or rural areas. U5CMSS of China has been described well in the other articles $(25,28,29)$.

\section{Data Collection}

Briefly, data collection in U5CMSS was based on the three-level administration network of women and children's health. It has also been well documented previously $(25,28,29)$. The order of data collection is village-township-county in rural areas, and communitysubdistrict-district in urban areas. The trained village doctor and director of the community committee collect the information on live births each month and report it to township and subdistrict $\mathrm{MCH}$ worker. Child death cases must be reported to the $\mathrm{MCH}$ worker at the township and subdistrict level within $3 \mathrm{~d}$ of the death. A structured household survey questionnaire about the deceased child should be administered by the $\mathrm{MCH}$ worker within $7 \mathrm{~d}$ through interviewing caregivers or attending nurses and physicians if the death occurred in hospital. Each quarter, the $\mathrm{MCH}$ worker reports the number of live births and child deaths to the county/district-level MCH institution after examination and approval. Then, the reports are reviewed and verified by county/district-level, prefectural and municipal level, and provincial level $\mathrm{MCH}$ institution. The national office for $\mathrm{MCH}$ surveillance finally confirms the reports and encodes the underlying cause of death for each case using International Classification of Diseases-10.

\section{Quality Control}

To ensure the accuracy of the data collected in U5CMSS, regular quality control was conducted at all levels of the surveillance networkquarterly at township and subdistrict levels, semiannually at the county level, and annually at prefectural and municipal levels, provincial level and national level at selected sites. The National Office randomly checked the quality of surveillance data from counties/districts in six to eight provinces every year. Quality control focuses on missing reports and erroneous causes of death by cross-examining 
multisource data from hospitals, the Center for Disease Control and Prevention, the Population and Family Planning Commission and the Civil Affairs Bureau. This is because data about live births and child deaths are collected by all these institutions. Thus, the under-reported rate for live births and deaths grouped as urban or rural was obtained for the adjustment of child mortality rate.

\section{Statistical Analysis}

Neonatal death is defined as death occurring less than $28 \mathrm{~d}$ after birth. The NMR and cause-specific NMRs were calculated as the numbers of deaths per 1,000 live births. NMR was further broken down into early neonate mortality rate (ENMR, $0-6 \mathrm{~d}$ of births) and late neonate mortality rate (7-27 $\mathrm{d}$ of births). As considering some annual numbers of cause-specific neonatal deaths were too low to precisely calculate the mortality rates, we aggregated the numbers of neonatal deaths and live births for each $3 \mathrm{y}$. Thus, the NMRs were arbitrarily aggregated into 3-y periods (1996-1998, 1999-2001, 2002-2004, 2005-2007, 2008-2010 and 2011-2013).

We used changing amplitude (\%) to measure the change in NMR, ENMR and late neonate mortality rate during the study periods. Changing amplitude is defined as $\left(\mathrm{NMR}_{2011-2013}-\mathrm{NMR}_{1996-1998}\right) /$ $\mathrm{NMR}_{1996-1998} \times 100 \%$. The RR and its $95 \% \mathrm{CI}$ of NMR between rural and urban areas for each period were calculated using a Poisson regression (30). As for the each cause-specific NMR, the RRs between rural and urban areas (and 95\% CIs) were also outputted from Poisson regression separately. The general form of the model was expressed as $\ln \left(\right.$ Deaths $\left._{\mathrm{t}}\right)=\ln \left(B_{\mathrm{t}}\right)+\alpha+\Sigma \beta_{1 \mathrm{i}} \times$ Period $_{\mathrm{i}}+\beta_{2} \times \mathrm{UR}+\Sigma \beta_{3 \mathrm{i}}$ $\times$ UR $\times$ Period $+\varepsilon$, where Death is the number of neonatal death (or cause-specific neonatal death) in the period of t. $B_{\mathrm{t}}$ is the number of live births. Period are dummy variables for five periods (1999-2001, 2002-2004, 2005-2007, 2008-2010, and 2011-2013) and the period of 1996-1998 was set as reference group. UR is the categorical variable indicating urban or rural (UR $=0$ refers to urban; $\mathrm{UR}=1$ refers to rural). Therefore, the RR for the period of 1996-1998 was calculated by the logarithm of $\beta_{2}$, and RR for others periods were separately calculated by the logarithm of $\left(\beta_{2}+\beta_{3 \mathrm{i}}\right)$. The $95 \%$ CIs of RRs were yielded on the basis of the standard deviation of $\beta_{2}$ or $\left(\beta_{2}+\beta_{3 \mathrm{j}}\right)$. Besides, $95 \%$ CIs for each cause-specific NMR for each period were obtained from the same models. For the urban area $(\mathrm{UR}=0)$, the $95 \%$ CIs of the number of neonatal deaths were outputted from the

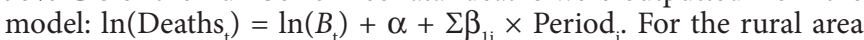
$(\mathrm{UR}=1)$, they were outputted from the model: $\ln ($ Deaths $)=\ln \left(B_{\mathrm{t}}\right)+$ $\alpha+\beta_{2}+\left(\Sigma \beta_{1 \mathrm{i}}+\Sigma \beta_{3 \mathrm{i}}\right) \times$ Period. In addition, the trend analysis of the proportion of neonatal deaths due to a specific cause with periods was performed using the Cochran-Armitage trend test (31).

All statistical tests were performed using SAS software (SAS version 9.0; SAS Institute, Cary, NC). A statistically significant difference was defined as $a=0.05$.

Ethical approval for our study was provided by the Ethics Committee of West China Second University Hospital, Sichuan University, China (grant number: 2010015). The oral consent was obtained from the guardian of each neonatal death for the epidemiology study.

\section{ACKNOWLEDGMENTS}

The authors thank all the healthcare workers involved in the data collection process for their efforts in providing data and investigating cases. Ruifeng $L u$ : Lu conducted the literature review and analyses and drafted the manuscript, and approved the final manuscript as submitted. Xiaohong Li and Sufang Guo: Li and Guo contributed to the interpretation of results and drafting of sections of the discussion, and approved the final manuscript as submitted. Huiqing Wang, Jun Zhu, Chunhua He, Qi Li, and Leni Kang: They conducted the data collection and analysis, and critically reviewed the manuscript, and approved the final manuscript as submitted. Jun Tang and Yanping Wang: Both contributed to the design of the study and interpretation of results, and approved the final manuscript as submitted. Dezhi Mu: The author was a senior author and supervised the design of the study, and approved the final manuscript as submitted.

\section{STATEMENT OF FINANCIAL SUPPORT}

This work was supported and funded by UNICEF (grant YH601-11-1141), National Nature Scientific Foundation of China (grant 8133016), the Health
Ministry of China (grants QT2003-009 and 05wsb-02), Science and Technology Bureau of Sichuan Province (2014SZ0149), and Program for Changjiang Scholars and Innovative Research Team in University (IRT0935).

Disclosures: The authors have no other funding or conflicts of interest to disclose.

\section{REFERENCES}

1. Wang H, Liddell CA, Coates MM, et al. Global, regional, and national levels of neonatal, infant, and under-5 mortality during 1990-2013: a systematic analysis for the Global Burden of Disease Study 2013. Lancet 2014;384:957-79.

2. Feng J, Yuan XQ, Zhu J, et al. Under-5-mortality rate and causes of death in China, 2000 to 2010. Zhonghua Liu Xing Bing Xue Za Zhi 2012;33:558-61.

3. Rudan I, Chan KY, Zhang JS, et al.; WHO/UNICEF's Child Health Epidemiology Reference Group (CHERG). Causes of deaths in children younger than 5 years in China in 2008. Lancet 2010;375:1083-9.

4. Feng XL, Theodoratou E, Liu L, et al. Social, economic, political and health system and program determinants of child mortality reduction in China between 1990 and 2006: a systematic analysis. J Glob Health 2012;2:010405.

5. Health Ministry of China. Health Statistical Yearbook of China, 1st edn. Beijing, China: Peking Union Medical College press, 2013:201.

6. Yanping W, Lei M, Li D, et al. A study on rural-urban differences in neonatal mortality rate in China, 1996-2006. J Epidemiol Community Health 2010;64:935-6.

7. Thea D, Qazi S. Neonatal mortality -4 million reasons for progress. Lancet 2008;371:1893-5.

8. Wang J, Wang P, Wang X, Zheng Y, Xiao Y. Use and prescription of antibiotics in primary health care settings in China. JAMA Intern Med 2014;174:1914-20.

9. Chamberlain M. A Baby-Friendly Hospital initiative in northern China. Nurs Ethics 1997;4:511-8.

10. Liang J, Li X, Dai L, et al. The changes in maternal mortality in 1000 counties in mid-Western China by a government-initiated intervention. PLoS One 2012;7:e37458.

11. Li X, Chongsuvivatwong V, Sangsupawanich P, Xia X. Evaluation of short term integrated management of childhood illness training on the clinical competency of village doctors in Yunnan, China. J Med Assoc Thai 2012;95:890-4.

12. Zhang Y, Dai Y, Zhang S. Impact of implementation of Integrated Management of Childhood Illness on improvement of health system in China. J Paediatr Child Health 2007;43:681-5.

13. Feng XL, Guo S, Hipgrave D, et al. China's facility-based birth strategy and neonatal mortality: a population-based epidemiological study. Lancet 2011;378:1493-500.

14. National Health and Family Planning Commission of the People's Republic of China. China Statistical Yearbook of Health and Family Planning, 1st edn. Beijing, China: Peking Union Medical College press, 2014:217.

15. Xu T, Wang $\mathrm{H}$, Gong $\mathrm{L}$, et al. The impact of an intervention package promoting effective neonatal resuscitation training in rural China. Resuscitation 2014;85:253-9.

16. Lewis G. Beyond the numbers: reviewing maternal deaths and complications to make pregnancy safer. Br Med Bull 2003;67:27-37.

17. Manasyan A, Chomba E, McClure EM, Wright LL, Krzywanski S, Carlo WA; Eunice Kennedy Shriver National Institute of Child Health and Human Development Global Network for Women's and Children's Health Research. Cost-effectiveness of essential newborn care training in urban first-level facilities. Pediatrics 2011;127:e1176-81.

18. Mwansa-Kambafwile J, Cousens S, Hansen T, Lawn JE. Antenatal steroids in preterm labour for the prevention of neonatal deaths due to complications of preterm birth. Int J Epidemiol 2010;39:Suppl 1:1122-33.

19. Lawn JE, Mwansa-Kambafwile J, Horta BL, Barros FC, Cousens S. 'Kangaroo mother care' to prevent neonatal deaths due to preterm birth complications. Int J Epidemiol 2010;39:Suppl 1:i144-54.

20. Klein LL, Gibbs RS. Use of microbial cultures and antibiotics in the prevention of infection-associated preterm birth. Am J Obstet Gynecol 2004;190:1493-502. 


\section{Articles | Luetal.}

21. Bassani DG, Roth DE. China's progress in neonatal mortality. Lancet 2011;378:1446-7.

22. Kadaga R, da Silva JB Jr, Featherstone L. Caring for preterm babies is a test of how we respond to our most vulnerable citizens. Lancet 2013;382:1613-4.

23. Liu L, Oza S, Hogan D, et al. Global, regional, and national causes of child mortality in 2000-13, with projections to inform post-2015 priorities: an updated systematic analysis. Lancet 2015;385:430-40.

24. World Health Organization. Sixty-third World Health Assembly (A63/10): Birth defects, Report by the Sectetariat, 2010. (http://apps.who.int/gb/ ebwha/pdf_files/WHA63/A63_10-en.pdf) Accessed 4 February 2016.

25. Wang YP, Miao L, Dai L, et al. Mortality rate for children under 5 years of age in China from 1996 to 2006. Public Health 2011;125:301-7.

26. Mathers C. Ending preventable child deaths: addressing the high-risk days after birth. Lancet Glob Health 2014;2:e617-8.
27. Glass RI, Guttmacher AE, Black RE. Ending preventable child death in a generation. JAMA 2012;308:141-2.

28. Liang J, Mao M, Dai L, et al. Neonatal mortality due to preterm birth at 28-36 weeks' gestation in China, 2003-2008. Paediatr Perinat Epidemiol 2011;25:593-600.

29. Wang Y, Zhu J, He C, Li X, Miao L, Liang J. Geographical disparities of infant mortality in rural China. Arch Dis Child Fetal Neonatal Ed 2012;97:F285-90.

30. Li X, Zhu J, Dai L, et al. Trends in maternal mortality due to obstetric hemorrhage in urban and rural China, 1996-2005. J Perinat Med 2011;39: 35-41.

31. He C, Kang L, Miao L, et al. Pneumonia mortality among children under 5 in China from 1996 to 2013: an analysis from National Surveillance System. PLoS One 2015;10:e0133620. 